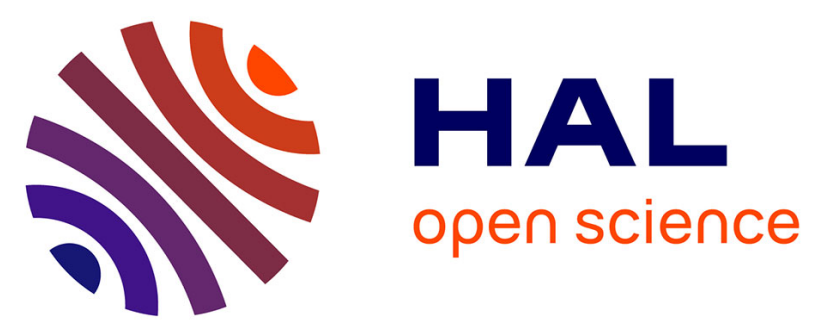

\title{
249th ENMC International Workshop: The role of brain dystrophin in muscular dystrophy: Implications for clinical care and translational research, Hoofddorp, The Netherlands, November 29th-December 1st 2019
}

Jos G.M. Hendriksen, Mathula Thangarajh, Hermien E Kan, Francesco Muntoni, Dr y Aoki, Dr P Collin, Dr M Colvin, Dr N Doorenweerd, Prof A Ferlini, Dr A Goyenvalle, et al.

\section{- To cite this version:}

Jos G.M. Hendriksen, Mathula Thangarajh, Hermien E Kan, Francesco Muntoni, Dr y Aoki, et al.. 249th ENMC International Workshop: The role of brain dystrophin in muscular dystrophy: Implications for clinical care and translational research, Hoofddorp, The Netherlands, November 29thDecember 1st 2019. Neuromuscular Disorders, 2020, 30 (9), pp.782-794. 10.1016/j.nmd.2020.08.357 . hal-03009729

\section{HAL Id: hal-03009729 \\ https://cnrs.hal.science/hal-03009729}

Submitted on 2 Dec 2020

HAL is a multi-disciplinary open access archive for the deposit and dissemination of scientific research documents, whether they are published or not. The documents may come from teaching and research institutions in France or abroad, or from public or private research centers.
L'archive ouverte pluridisciplinaire HAL, est destinée au dépôt et à la diffusion de documents scientifiques de niveau recherche, publiés ou non, émanant des établissements d'enseignement et de recherche français ou étrangers, des laboratoires publics ou privés. 
This is the accepted draft copy of the article:

Neuromuscul Disord. 2020 Sep;30(9):782-794.

doi: 10.1016/j.nmd.2020.08.357. Epub 2020 Aug 15. PMID: 32912717

\section{9th ENMC International Workshop: The role of brain dystrophin in muscular dystrophy: Implications for clinical care and translational research, Hoofddorp, The Netherlands, November 29th-December 1st 2019}

Jos G M Hendriksen 1 , Mathula Thangarajh 2 , Hermien E Kan 3 , Francesco Muntoni 4 , ENMC 249th workshop study group (see below the list of all co-authors and affiliations)

ENMC 249th workshop study group:

Dr Y Aoki 5, Dr P Collin 6, Dr M Colvin 7, Dr N Doorenweerd 6, Prof A Ferlini 8, Dr A Goyenvalle 9, Dr J J Hendriksen 6, Dr J Hoskin 10, Dr H E Kan 6, Mr F Lamy 9, Dr K Maresh 10, Prof F Muntoni 10, DrE H Niks 6, Prof U Schara 11, Prof D Skuse 10, Prof V Straub 10, Prof S Takeda 5 , Dr M Thangarajh 7, Dr N Truba 7, Prof S Tyagarajan 12, Dr C Vaillend 9, Dr M van Putten 6, Prof J Vissing 13, E Vroom 6

\section{Affiliations}

1 Kempenhaeghe Center For Neurological Learning Disabilities, Heeze, the Netherlands; Duchenne Center, the Netherlands.

2 Department of Neurology, Virginia Commonwealth University, Richmond, USA.

3 Duchenne Center, the Netherlands; C.J. Gorter Center for High Field MRI, Department of Radiology, Leiden University Medical Center, Leiden, the Netherlands. Electronic address: h.e.kan@lumc.nl. 4 The Dubowitz Neuromuscular Centre, University College London, Great Ormond Street Institute of Child Health, London, UK; NIHR Great Ormond Street Hospital Biomedical Research Centre, London, UK.

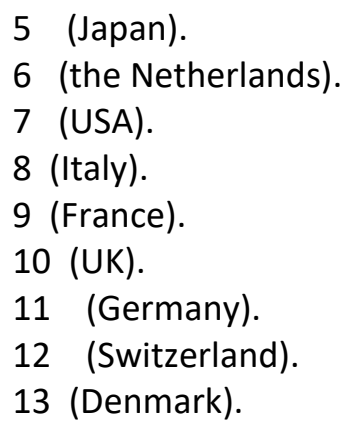




\section{9th ENMC workshop:}

The role of brain dystrophin in muscular dystrophy: Implications for clinical care and translational research, Hoofddorp, the Netherlands, November 29 $9^{\text {th }}-$ December $1^{\text {st }} 2019$

Jos G.M. Hendriksen ${ }^{1,2}$, Mathula Thangarajh ${ }^{3}$, Hermien E. Kan ${ }^{2,4^{*}}$, Francesco Muntoni ${ }^{5,6}$, on behalf of the ENMC $249^{\text {th }}$ workshop study group $\$$

${ }^{1}$ Kempenhaeghe Center for neurological learning disabilities, Heeze, the Netherlands

${ }^{2}$ Duchenne Center the Netherlands ${ }^{3}$ Department of Neurology, Virginia Commonwealth University, Richmond, USA ${ }^{4}$ C.J. Gorter Center for High Field MRI, Department of Radiology, Leiden University Medical Center, Leiden, the Netherlands

${ }^{5}$ The Dubowitz Neuromuscular Centre, University College London, Great Ormond Street Institute of Child Health, London, UK ${ }^{6}$ NIHR Great Ormond Street Hospital Biomedical Research Centre, London, UK

\# corresponding author

\section{Correspondence to:}

Hermien E. Kan, PhD

Leiden University Medical Center

C.J. Gorter Center for high field MRI, Department of Radiology 
PO Box 9600, 2300 RC Leiden

The Netherlands

Email: $\underline{\text { h.e.kan@lumc.nl }}$

Phone: +31 715266097 Fax: +31 715248256

\section{ORCID IDs}

J. Hendriksen: 0000-0002-3930-7697; M. Thangarajh: 0000-0001-9811-0838; H.E. Kan: 0000-0002-5772-7177; F. Muntoni: 0000-0002-9102-5232

\section{Conflict of interest statements}

JGMH has nothing to disclose, MT reports reports grants from American Association of Neuromuscular \& Electrodiagnostic Medicine/American Neuromuscular Foundation during the conduct of the study; HEK reports consultancy from PTC therapeutics and trial support from ImagingDMD, outside the submitted work. No personal fees were received, all payments go the LUMC. FM reports reports personal fees from Scientific advisory boards for Sarepta, Santhera and Pfizer during the conduct of the study; . 


\section{Highlights}

- Overview of the multiple dystrophin isoforms expressed in the brain

- Large body of preclinical data and possibilities for postnatal dystrophin restoration

- Overview of neurocognitive and behavioral heterogeneity in DMD and BMD

- Neuromuscular specialists to also focus on early diagnosis and intervention for brain comorbidities

- Need to develop standard operation procedures for neurocognition and behavior assessment 


\section{Introduction}

The 249th ENMC workshop was held from the 29th of November to the 1st of December 2019, and brought together 24 representative of patients, neuromuscular, neuropsychology and psychiatry experts from Europe, the United States and Japan. The topic of this workshop was the brain co-morbidities in Duchenne and Becker muscular dystrophies (DMD and BMD respectively).

Following a welcome from Alexandra Breukel, ENMC representative and director, and the chairpersons of the workshop, Jos Hendriksen, Mathula Thangarajh, Hermien Kan and Francesco Muntoni, Francesco Muntoni gave an overview of the topic.

The molecular basis of the underlying brain involvement in Duchenne muscular dystrophy (DMD) and in Becker muscular dystrophy (BMD) is increasingly being understood. Better understanding of the cognitive and behavioural, psychiatric comorbid symptoms that may affect a significant proportion of boys and men with DMD (up to $50 \%$ ) and BMD has important clinical implications for the affected individuals and also for the understanding of the biological function(s) of the various dystrophin isoforms in the brain. These comorbidities are acknowledged in the 2018 DMD Clinical Care guidelines (Standards of care [1]]) and recommendations are described for psychosocial care i.e. routine mental health screening and neuropsychological testing. Standard assessment operating procedures are not yet described except for recommendation for using some general screening tools on depression, anxiety, and psychosocial adjustment. More broadly, there is the need to better understand (i) the role(s) of the multiple dystrophin isoforms expressed in the central nervous system, (ii) their interacting partners, (iii) their contribution to the various comorbidities that characterise individuals with $\mathrm{DMD}$ and $\mathrm{BMD}$, and (iv) the potential for postnatal dystrophin restoration in addressing some of these comorbidities. While DMD 
experimental therapies currently in clinical trials only improve skeletal muscle function and hence do not comprehensively address the unmet brain-related comorbidities and needs of this patient population, recent preclinical work suggests that at least some of the pathological behavioural features of the $m d x$ mouse can be improved by postnatal brain dystrophin restoration. These various issues were at the core of a multidisciplinary workshop sponsored by the ENMC in which clinical, preclinical and translational research aspects of brain involvement in DMD and BMD were discussed.

\section{Dystrophin gene regulation and protein expression in the human and murine}

brain: Alessandra Ferlini, Ferrara, Italy.

Dr. Ferlini provided an overview of the genomic organisation of the $D M D$ locus, which spans 2.2 Mb on Xp21, contains 79 consecutive exons, and many alternatively spliced exons (either novel or constitutive) (table 1)[2].

Three promoters (muscle, cerebral and Purkinje or Dp427m, Dp427c, Dp427p) each with their unique first exon, drive the corresponding full-length isoforms which show high tissue specificity. Four shorter 3' isoforms are transcribed by specific promoters and their first exons (Dp260, Dp140, Dp116, Dp71), and are tissue-specific or developmentally regulated. Pathogenic mutations in the $D M D$ gene, mainly deletions (65\%), duplications (10\%) and small mutations (25\%), cause DMD and BMD. Very accurate mutation detection is currently available via MLPA (multiplex ligationdependent probe amplification) or full sequencing, mainly using next generation sequencing methods [11]. DMD encodes the dystrophin protein, a sub-sarcolemma protein involved in sarcolemma integrity, force transduction, muscle signalling and regeneration. The $D M D$ transcript has a very complex transcription process, mediated by the 7 promoters, at least 20 described alternative splicing events, 
recursive splicing (non-sequential exon splicing), and 2 different polyA sites in the extreme long 3'UTR, which is also the target of several miRNAs [12]. The locus also generates several long ncRNAs, which amount inversely correlates with the mRNA quantity in skeletal muscle, therefore probably having a transcriptional repression role. $D M D$ transcripts are tissue-specific (skeletal and cardiac muscle, and the brain) as is the dystrophin protein translation. However, suboptimal and very low transcriptional levels of the $D M D$ mRNA are detectable by highly sensitive techniques (as RNA-seq). In brain, $D M D$ transcripts are detectable in cerebral cortex, cerebellum, hippocampus, amygdala, basal ganglia, hypothalamus and thalamus, and spinal cord (Human Protein Atlas, www.proteinatlas.org). The Dp140 and Dp71 isoforms are known to play a role, still not fully deciphered, in the brain and consequently in cognitive impairment frequently occurring in DMD boys. The Dp140 locus architecture is extremely complex and unusual for Homo Sapiens [13]. Its promoter and unique first exon are located in intron 44, this unique first exon embedded in the Dp140 promoter region includes the putative TATA-like and CAAT boxes. This first exon splices directly to the constitutive exon 45 canonical acceptor splice site and then follows the splicing behaviour of the full-length $D M D$ transcript. Nevertheless, this large region remains untranslated, and the ATG start site locates within the constitutive exon 51 ; therefore, the Dp140 protein translation starts here, and completely overlaps to the 3' end of the dystrophin protein. This spliced, long 5'UTR of Dp140 makes this region a target for post-transcriptional regulatory events, as those mediated by miRNA, Dicer complex, or any other machinery involved in post-splicing regulation. In general, $D M D$ introns are poorly studied, and almost never investigated in patients. The new approach based on whole genome sequencing will certainly help us in identifying intronic regulatory regions, as, for 
example, the one located in intron 62 which contains a(GAA)n triplet repeat the function of which is unknown. Studying DMD mRNA has allowed to unequivocally demonstrate the phenomenon of the 5'-3' transcript imbalance, meaning a reduction of the 3' end of the mRNA [14]. This occurs physiologically in normal muscle but is more pronounced in DMD patients' muscle. Very little is known about $D M D$ locus epigenetics. A few studies demonstrated the presence of regions of open chromatin structures, which may have still unravelled regulatory roles [15]. Finally, it should not be forgotten that the $D M D$ gene is an onco-suppressor gene, acting either in myogenic cancers but also in brain cancer types [16].

\section{Temporal and spatial expression of dystrophin in the brain: Nathalie} Doorenweerd, Newcastle UK and Leiden the Netherlands.

Dr. Doorenweerd summarised the information available on the expression of the various DMD isoforms in the adult brain and during development [17]. In human brain, the full-length isoforms Dp427m and Dp427c, are present throughout the cortex and basal ganglia where they gradually accumulate with age. Dp427p is almost absent in the human brain, in contrast to mice. Dystrophin has the highest expression in the hippocampus and amygdala and lowest in the cerebellum. With respect to the shorter isoforms: early studies already indicated that Dp140 is expressed predominantly during human brain development, which was confirmed and expanded upon by a recent study from Doorenweerd et. al. that identified its high expression in fetal brain but continued presence throughout adulthood [17]. There is little insight in the role(s) or binding partners of Dp140. However, the analysis of genes temporally and physically co-expressed with Dp140 revealed an enrichment in gene ontology terms related to early neurodevelopmental processes, dendrite development and axonal 
guidance. Absence of Dp140 may lead to abnormal nervous system tract morphology, in keeping with the altered white matter microstructure detected by MRI in some DMD patients [18].

Mutations affecting Dp71 are rare ( $\sim 5 \%$ of all mutations) and lead to severe intellectual disability. Dp71 is ubiquitously expressed in the brain, where it potentially has a role in glutamergic transmission and in glial-dependent extracellular ion homeostasis. Analyses identified that the genes that are co-expressed with Dp71 were enriched in gene ontology terms related to receptor binding as well as to vascular development. A similar gene ontology analysis of Dp427 resulted in enrichment for genes involved in neuronal function, synaptic membrane organisation and channel activity. Recent data indicate a more complex pattern of expression of dystrophin in different cell types, which include, in addition to neurons, astrocytes; oligodendrocytes [19], and endothelial cells [20].

Dr. Doorenweerd also discussed that a significant wealth of information is now available in the various databases. At the same time, fundamental questions regarding the precise expression of the different isoforms during different developmental stages, and the analysis of specific subpopulation of cells will require additional wet lab work, including single cell RNA sequencing, to decipher some of the complexity in the normally developing brain. The next question could be how the lack of dystrophin affects these different processes.

Neural correlates of neuropsychiatric disorders: David Skuse, London, UK.

Dr. Skuse presented data from different studies on the effects of dystrophin on brainrelated functioning. Data suggest that Dp140 loss may be involved in Autism 
Spectrum Disorders (ASD) and that Dp71 loss may be involved in severe cognitive impairment. The use of rating scales in assessment of behaviour problems in DMD was critically discussed as these instruments have shown limited reliability and validity according to a recent review study on all the different instruments-61 in total—being used in DMD research [21]. Data from his earlier research work were presented that highlighted the relationship between autistic traits, anxiety and amygdala dysfunction in Turner syndrome. Dr. Skuse suggested that a novel paradigm could be used for the assessment of neuropsychiatric characteristics associated with DMD, by utilizing our knowledge about the neural processes involved in fear conditioning. He argued that dysfunction in these processes could underlie some of the neuropsychiatric characteristics of DMD. It is known that dystrophin is highly expressed in the amygdala, cerebellum and prefrontal cortex. Loss of dystrophin may disrupt neuronal (GABAA-mediated) signalling in the amygdala. Impaired fear conditioning in mouse models of DMD can be modified by pharmacological interventions, raising the prospect that this novel research paradigm could prove to be of value in human intervention studies too.

The role of dystrophin in the brain: GABA and other postsynaptic functions: Shiva Tyagarajan, Zurich, Switzerland

Dr Tyagarajan's presentation was focused on the diversity of GABAergic inhibitory connectivity and synaptic proteins in the brain and the role that the dystrophin glycoprotein complex (DGC) has in regulating this diversity. The DGC complex has indeed a crucial role not only for muscle structural integrity but also for the synaptic organization in brain [22]. 
In the cerebral cortex, hippocampus and cerebellum, the DGC (specifically the DGC formed by the full-length Dp427 isoforms), colocalizes with a subset of GABAA receptors (GABAAR). GABAARs are heteropentamers, and those enriched at the post synapse are most commonly composed of the $2 \alpha, 2 \beta$, and $y$ subunits. Some of the postsynaptic specialization of GABAARs is conferred by the a subunit, with a1 containing receptors enriched on the dendrites and soma, and a2 containing receptors enriched on the soma and axonal initial segment. Further complexity is added due to brain circuits relying on unique mechanisms to control synapse targeting, specificity and molecular specialization. The DGC plays an important role in the molecular specialization of GABAAR.

In order to address the molecular players with which the DGC interacts in the brain, the group of Tyagarajan has used mice that specifically lack neuronal dystroglycan. In these mice, a subset of GABAergic synapse is lost on CA1 hippocampal pyramidal neurons. The loss of dystroglycan in pyramidal cells has little effect on GABAAR expression and other glutamatergic synaptic proteins. However, the absence of dystroglycan expression led to loss of CCK-positive GABAergic interneuron terminals (CCK8 is a molecular marker of GABAAR). Hence, molecular diversity in postsynaptic compartment allows for the specific recruitment of CCK-positive interneuron by dystroglycan on pyramidal cell soma. Mice with dystroglycan-dependent loss of CCK terminals exhibit defects in electrophysiological theta oscillations, which are considered to contribute to memory and cognition defects.

Building on these results highlighting the synaptic heterogeneity of DGC, the group of Tyagarajan has recently identified a new extracellular matrix protein, Adamtsl3 as a ligand for alpha-dystroglycan. Immunolocalisation studies demonstrate indeed that Adamts|3 localises with dystroglycan at the GABAAR. Adamtsl3 has a distinct 
expression pattern; with high expression in pyramidal cells of CA3, 2, 1 regions. By targeted inactivation of Adamtsl3, the DGC assembly is impaired, leading to loss of CCK-positive GABA terminals. Adamts/3Ko mice exhibit memory defects, poor balance, decrease anxiety behaviour and hyperactivity. These novel findings offer yet another perspective on the molecular partners of the DGC in the brain and how the defect of a protein within this complex can lead to intellectual disability. It will now be of interest to perform reciprocal studies and assess the extent of mislocalisation of Adamts 13 in the dystrophin-deficient brain, but also in the brain of the various muscular dystrophies in which the defective glycosylation of alpha dystroglycan leads to a loss of binding with various extracellular ligands.

Dystrophin networks in the brain of $\boldsymbol{m d x}$ mice. Yoshitsugu Aoki, Tokyo, Japan.

Dr. Yoshitsugu's presentation was focused on the analysis of the similarities and dissimilarities in behavioural responses between the commonly studied $m d x$ mouse, carrying a nonsense mutation in exon $23(m d x 23)$ that disrupts expression of the Dp427 full-length isoforms, and the $m d x 52$ mouse, in which a deletion of the murine exon 52 leads to disruption of both Dp427 and Dp140.

Dr. Yoshitsugu initially discussed the similarities between the two models regarding the threat and stress responses. In $m d x 23$, the deficit of brain dystrophin has an impact on unconditioned and conditioned defensive behaviour. Apart from the murine studies, there is evidence that dystrophin deficiency affects defensive behaviours and fear responses in other animals. In quails, dystrophin was recently identified in the genomic region responsible for a fear-related behaviour, namely tonic immobility [23]. In commercial porcine populations, polymorphisms in the porcine $D M D$ gene and 
decreased dystrophin expression have been associated with a novel stress response syndrome, sometimes resulting in death during regular handling, and transport for slaughter, or following anaesthesia. A brain structure considered to be important in this pathological response is the amygdala, and in particular the basolateral ganglia, where the role of Dp427 in organising the postsynaptic GABAAR has been previously described [23]. Dr. Yoshitsugu reported that the gross anatomy of the amygdala in the $m d x 52$ model is apparently normal; however the density of the GABAAR is reduced even further in $m d x 52$ compared to $m d \times 23$ mice. The reduction appears to predominantly affect the GABAA1 rather than the GABAA2 receptors. Patch clamp experiments on amygdala slices confirmed excitation/inhibition imbalance, greater in the $m d x 52$ compared to $m d x 23$. Deep behavioural phenotyping of both $m d x 23$ and $m d x 52$ demonstrated differences between these two models, especially related to tasks on social behaviour.

Behavioural assessment of $\boldsymbol{m d x}$ mice: Cyrille Vaillend, Paris, France Dr. Vaillend focused on multidisciplinary studies in mouse models with a selective loss of distinct dystrophin-gene products to shed new light on the underlying brain role of dystrophins and to identify phenotypes that could be used as outcome measures in brain intervention studies. He focused on the $m d \times 23$ mouse lacking Dp427 and the Dp71-null mouse, with selective inactivation of Dp71, without involvement of the remaining isoforms.

Regarding $m d x 23$, his group demonstrated that these mice have severe emotional disturbances characterized by enhanced unconditioned fear, stress sensitivity and anxiety, which may reduce their locomotor activity, and therefore constitute a bias in preclinical study of treatments for muscular dystrophy that is important to be aware of 
[24]. He also demonstrated that altered emotional reactivity and changes in executive functions may contribute to the abnormal phenotype reported in social behaviour and communication. He further reported that the $m d \times 23$ mice also show selective deficits in long-term memory in various tasks including lever-press procedural learning, spatial memory, contextual and cued fear conditioning and recognition memory. These defects are not associated with global brain dysfunction, as other functions are preserved, including short-term and working memory, social memory, discrimination learning, behavioural flexibility and learning extinction. The $m d \times 23$ is characterised by reduced GABAergic function and clustering of postsynaptic GABAAR, altered synaptic plasticity and synapse morphology [25]. These GABA receptor cluster defects can remarkably be compensated postnatally with $\mathrm{Dmd}$ gene correction strategies based on exon-skipping, which was associated with normalization of synaptic plasticity and emotional reactivity in $m d x$ mice [26-28]. Different levels of brain dystrophin restoration could be achieved by the different strategies, the highest being using intracerebral injections of the AAV-U7 system, and lower levels with the systemic administration of Tricyclo DNA (tcDNA) antisense oligonucleotides (AONs).

Regarding the Dp71-null mouse, this strain exclusively lacks the abundant Dp71 isoform in the brain. Dp71 has been previously detected in glutamatergic synapses by the group of Dr. Vaillend [29], but it is also strongly expressed in perivascular astrocytes where its loss may alter brain ion homeostasis and contribute to altered neuronal excitability. Dr. Vaillend reported on the deep phenotyping of the Dp71-null model, and indicated that the behavioural profile of this mouse differed from that of $m d x 23$ mice, as these mice show deficits in short-term and working memories, spatial learning and cognitive flexibility, along with anxiety-like behaviours [29, 30]. Interestingly, this behavioural profile resembles the deficits reported in DMD patients 
holding mutations that impair Dp71 expression [31]. The lack of Dp71 in this mouse model was associated with enhanced glutamatergic synaptic transmission, imbalance in excitation/inhibition, impaired synaptic plasticity and altered synapse morphology $[29,30,32]$. These data suggest that mice holding distinct mutations in the Dmd gene display different profiles of cognitive and behavioural dysfunctions, which rely on the impaired expression of various dystrophins playing different functions in specific brain cell types. Both loss of Dp427 and Dp71 affect neuronal excitation but distinct neurotransmitter systems and brain networks are responsible for the deterioration of brain functions. Remarkably, genetic therapies in $m d x 23$ suggest that part of the behavioural disturbances associated with lack of Dp427 could be compensated with postnatal interventions based on rescued expression of the brain dystrophins. Similar ongoing studies in the exon 52-deleted $m d x 52$ mouse, which lacks Dp427 and Dp140 in brain, will shed new light on genotype-phenotype relationships and on the potential of exon-skipping strategies to rescue different brain dystrophins.

Animal models to evaluate dystrophin function in the brain: Maaike van Putten, Leiden, the Netherlands

Dr. van Putten summarised the various DMD animal models that have been described (dogs, pigs, cat (extinct), rats and mice), the only species that has been studied in detail regarding behaviour and/or brain pathology is the mouse. She reviewed the different $m d x$ mouse models available, which carry mutations spread over the Dmd gene and consequently lack one or multiple brain specific dystrophin isoforms. The mouse models on which publications are available are the $m d x$ mouse (lacking Dp427), the Dp71-null mouse (only lacking Dp71) and the $m d x^{3 \mathrm{cv}}$ mouse. This latter model expresses low dystrophin levels in muscle, but no detailed studies 
have been performed regarding brain dystrophin expression. Mouse models lacking both Dp427 and Dp140 are now also available $(m d x 52$, see the presentation of $\mathrm{Dr}$ Yoshitsugu; and $\left.m d x^{4 \mathrm{cv}}\right)$, but there is little published material on their brain pathology. Dr. van Putten stressed the importance to characterise further in detail the role of Dp140 in brain, as half of the DMD patient population-and also a proportion of BMD patients-is deficient in this isoform in the brain. Referring to RNA studies, she suggested that a direct link of Dp140 to brain-cell development was hypothesized since Dp140 is predominantly expressed in the human embryo and expression levels drop after birth [17]. Obviously, this information could have implications for the success of therapies that aim to postnatally restore expression of Dp140.

Dr. van Putten presented on a study conducted in the $m d x^{4 c v}$ model. Behavioural tests revealed that there is no clear deterioration in spatial, discrimination and reversal learning (Morris water maze and cognition wall test), working memory (Tmaze) and anxiety (light bulb test) compared to $m d x$ mice. These data also highlighted the importance of the generation of standard operating procedures for behavioural testing in DMD mouse models. Indeed, previously reported reversal learning deficit in $m d \times 23$ mice could not be replicated even when using automated home-cages [33].

Dr. van Putten showed data of bulk RNA-seq experiments of several brain regions (cortex, hippocampus and cerebellum) comparing the $m d x$ and $m d x^{4 \mathrm{cr}}$ models. Interestingly and to some extent surprisingly, the majority of differentially expressed genes was observed when the two $m d x$ mouse models were compared to each other, instead of the comparisons between each of these models and wildtype mice. This data could be due to previously published differences in abundance of specific 
cell-types between the models [34], and further highlights the importance of performing single-cell RNA sequencing analyses of the brain.

Dr. van Putten also discussed other variables that need to be considered when assessing the relevance of the $m d x$ mouse as preclinical model for DMD patients. One example relates to chronic corticosteroid use in DMD which is known to influence behaviour. However, the mouse experiments are performed in corticosteroid naïve animals, while the influence of corticosteroids in the behavioural response of the $m d x$ mice has never been addressed. In addition, recent experimental evidence indicates that there are differences both in terms of temporal expression of dystrophin isoforms between humans and mice, especially with Dp140 which in the mouse is expressed throughout life; and in the pattern of expression of individual isoforms in discrete brain regions (for example ,the Purkinje isoform Dp427p, expressed in the cerebellum of normal mice but not in the human). Dr. van Putten indicated ongoing experiments using human induced pluripotent stem cell (hiPSC) that could facilitate to unravel the role of each of the dystrophin isoforms in brain cell development and function.

\section{Antisense treatment to correct dystrophin-deficiency in the brain: Shin'ichi}

Takeda, Tokyo, Japan

Dr. Takeda summarised the original seminal work of his group related to the role of dystrophin in amygdala GABA circuitries and the effect of postnatal dystrophin restoration [23]. In their studies, the group of Dr. Takeda explored the role of restraint to examine the emotional responses of $m d x 23$ mice, as this is a well-established emotionally aversive stimulus. In this study, wild type and $m d x 23$ mice behaved differently, with all $m d x$ mice displaying a dramatic reduction of locomotor activity 
and a strong freezing response. This frozen posture persisted to different extents up to 30 minutes, and was only observed after the restrain procedure, not following simply moved from one cage to another. Another test explored was the sensitivity of $m d x 23$ mice to electrical foot shock, as an index of fear-motivated unconditioned defensive response. $M d \times 23$ mice demonstrated a freezing response immediately after foot shock, and when re-exposed to the apparatus, 24 hours later. This suggests an increase in both the conditioned and unconditioned defensive response in these mice.

In order to shed light on the molecular basis for this pathological behaviour, this group analysed in detail the hippocampus and amygdala, as they are well-known to be involved in these responses. These studies demonstrated abundant expression of dystrophin in the basolateral and the lateral nuclei of the amygdala in wild type, but not $m d \times 23$ mice (suggesting a role of the full-length dystrophin isoform). Following previous observations of a role in dystrophin in the postsynaptic organisation of GABAAR, they demonstrated a significant reduction of these receptors in the basolateral nucleus of the amygdala. This morphological abnormality was accompanied by electrophysiological evidence of reduced activity of the GABAergic synapses. Their group proceeded with intracerebroventricular (ICV) administration of a morpholino oligonucleotide designed to skip exon 23 , using an osmotic infusion pump that persistently released the drug solution for 1 week. The highest dystrophin levels were detected between week 5 and 7 post infusion. Importantly, this was accompanied by an improvement in the freezing response of the treated mice that coincided with the highest level of dystrophin restoration [23]. These experiments provided evidence that postnatal restoration of brain dystrophin expression was accompanied by functional recovery of at least some of the behavioural deficiency 
observed in $m d x$ mice, paving the way to future translational research studies in this field.

\section{Systemic antisense treatment to restore dystrophin in the brain of $\mathbf{m d x}$ mice:} Aurelie Goyenvalle, Paris, France

The group of Dr. Goyenvalle has a longstanding interest in translational research aspect of dystrophin deficiency, both in muscle, and in the brain. In the past few years, her group has worked with another antisense chemistry, the tcDNA, a novel class of AONs. In her studies, she was able to demonstrate that repeated systemic administration of tcDNA AONs during a period of 12 weeks, not only induced the skipping of exon 23 in muscle, but, by crossing the blood brain barrier, also rescued the expression of truncated but functional dystrophins in the cerebellum and hippocampus albeit at low levels $(\sim 2-3 \%)[27,35]$. Nevertheless the unconditioned fear response was completely normalized in the tcDNA treated $m d x 23$ mice [27]. These findings confirmed those of the previous study using the ICV morpholino AON administration mentioned above [23] regarding the potential reversibility of the freezing response. They also suggested that very low levels of Dp427 restoration obtained after systemic treatment, may be sufficient to restore these neurobehavioural features. The group of Dr. Goyenvalle is further investigating the cognitive defects in the $m d x$ mouse in order to determine the amount of dystrophin required to restore various aspects of the behavioural phenotype. This is now being pursued not only following systemic delivery of tcDNA AON, but also their administration after ICV injections. Using these different modalities of administration and different dosage and regimens, her group wishes to validate the effectiveness of locoregional treatment, and thus define the therapeutic threshold and the best time window. In order to establish these 
parameters, exon skipping levels are measured in several brain structures (hippocampus, cortex and cerebellum) as well as Dp427 restoration by western blot and immunohistochemistry at different time points after injection. The functional and behavioural effects of these different modalities of $\mathrm{AON}$ administration, including additional chemical modification to the tcDNA chemistry, is being done in collaboration with the group of Dr. Vaillend. Specific neurobehavioural tests have been selected to evaluate the reversibility of learning and memory deficits reported in the $m d x 23$ mouse model. Dr. Goyenvalle also reported recent data obtained in the mdx52 mouse following the systemic delivery of AON using the AAV9/U7 system, to induce exon 51 skipping. A single intravenous injection of AAV9/U7 induces efficient and sustained expression of Dp427 in skeletal muscles leading to an improvement of the muscle function in the treated $m d x 52$. However, despite low levels of exon 51 skipping measured in the brain $(\sim 5 \%)$, the dystrophin protein could not be detected, in line with a lack of improvement of their abnormal unconditioned fear response [36].

\section{Longitudinal assessment of brain involvement in $\mathbf{m d x}$ mice: Volker Straub,} Newcastle, UK

Dr. Straub presented data from their longitudinal study in $m d x 23$ mice looking at the mouse brain by MRI and by cognitive function tests [37]. The overall aim of their study was to develop techniques to non-invasively monitor cognition and brain function in $m d x 23$ mice over time. They studied 8 wild type and $8 m d x 23$ males from 4-18 months of age. All mice underwent brain MR imaging and cognitive function testing at 4, 6, 12 and 18 months of age. The application of MRI included T2-weighted fast spin-echo images and T1-weighted spin-echo images. For each mouse, axial, sagittal and 
coronal images were acquired. MRI identified a total brain volume increase in control mice over time $(p<0.05)$; but the $m d \times 23$ mice total brain volume increased significantly more $(p<0.01)$. Moreover, morphological changes in the $m d x 23$ brain ventricular system were identified, with abnormalities progressing with age. To monitor morphological brain changes between wild type and $m d \times 23$ mice over time, they applied voxel-based morphometry (VBM), which describes the automated process of analysing morphological differences between images of the brain by performing voxelwise statistics comparing groups of animals. They identified numerous changes with VBM in the $m d \times 23$ mouse brain and could show that the complexity of these changes increased between 6 and 18 months of age. Interestingly, the $m d x 23$ mice demonstrated enlargements in grey matter volume in the lower layers of the cortex, amygdala, and caudate putamen, in addition to reduced grey matter volume in the cerebellum, and hippocampus, regions known to be dystrophin rich, where the decrease in grey matter volume of these regions increased with age. The increased grey matter volume in the amygdala may be linked to the increased anxiety related behaviour, which the researchers observed in older $m d x 23$ mice. The changes identified by VBM in grey matter volume in various regions of the $m d x$ mouse brain were most noticeable from 12 months onwards. These findings matched those from the cognitive function tests focussing on learning and memory, for which the group used Barnes maze testing and the Novel Object Recognition test [38]. In the $m d x 23$ mice, the parameters measured by the Barnes maze test (success score, number of errors, time spent in the target quadrant) were affected more by age than in wild type mice. In particular, the time spent in the target quadrant, during short-term memory probe trial, was significantly reduced between 4 and 12 months of age, as was the short-term memory. The long-term memory of $m d x 23$ mice appeared to show the 
highest deficit between 4 and 12 months of age compared to long-term memory defect of control mice. In the Novel Object Recognition test mdx23 mice displayed a preference for the familiar object, and were opposed to the novel object, at all-time points, which may suggest that $m d x 23$ mice have an impaired recognition memory. Overall, the results from the longitudinal imaging and cognitive function tests suggested that there is a progressive decline in brain function with age in $m d x 23$ mice.

Neuropsychological manifestations in DMD (general intelligence, neurodevelopmental, emotional and behavioural symptoms): Kate Maresh, London, UK

Dr. Maresh presented data from a meta-analysis of CNS co-morbidities in DMD and BMD, and the results from a novel study of fear responses in young males with DMD. Although the fear response in DMD mouse models is commonly investigated, there is very limited information regarding the fear response in DMD boys, and the correlation with individual mutations affecting specific dystrophin isoforms. In order to address this issue, Dr. Maresh investigated whether abnormal fear responses were present in boys with DMD. She tested 31 DMD and 25 control males aged $7-12$ years using a bespoke fear conditioning task, specifically designed for this study and adapted for use in DMD boys. Participants were presented with trials of two neutral visual stimuli: one 'safe' stimulus presented alone; one paired with an aversive noise 'threat' to enable acquisition of a conditioned physiological fear response (skin conductance response, $\mathrm{SCR}$, and heart rate, HR). Both stimuli were later presented without the threat stimulus to assess extinction of the conditioned responses: skin conductance and heart rate. In this study, Dr. Maresh assessed the initial unconditioned response to threat and the 
conditioned responses during acquisition and extinction. She found that the initial (unconditioned) SCR to threat was significantly greater in DMD boys than controls $(p=0.041)$, and a trend of greater fear-induced bradycardia in DMD. Both DMD and Control groups acquired conditioned responses similarly, showing significant discrimination between threat and safe stimuli $(p=0.55)$ and both later extinguished the responses although in the DMD group the responses were non-significantly greater to the threat cue in the DMD than control group.

Only 20/28 DMD (71\%) participants completed more than 15/40 extinction trials due to drop-out. Dr. Maresh observed that DMD participants found the task more challenging than controls, where there were no dropouts.

The result of this pilot study, the first to investigate fear responses in DMD boys, is highly suggestive of exaggerated unconditioned responses to threat in these affected boys, providing similarities to the fear responses seen in $m d \times 23$ mice [24]. Further studies will be required to establish the contribution of the different dystrophin isoforms to this pathological behaviour, which could potentially become a useful objective measure in clinical trials of central nervous system (CNS)-targeted dystrophinrestoration therapies.

Dr. Maresh also presented the results of a meta-analysis she conducted of CNS comorbidities, updating the last meta-analysis in DMD from 2001 [39] and providing the first meta-analysis of these conditions in BMD. The meta-analysis included 4202 DMD and 420 BMD cases from 52 studies with direct assessment of cognitive and/or other CNS co-morbidities. Individual data including genotype and age was available for 513 DMD and 85 BMD cases. In DMD ( $n=20$ studies), mean full scale intelligence quotient (IQ) was $86.1(95 \% \mathrm{Cl} 84.8,87.4)$, verbal IQ $85.9(84.0,87.8)$ and performance IQ 89.5 
(86.6, 92.3). In BMD ( $n=7$ studies), mean full scale IQ was $88.3(95 \% \mathrm{Cl} 85.4,91.3)$, verbal IQ $91.1(82.3,99.8)$ and performance IQ $86.6(76.6,96.6)$. In DMD, the prevalence of autism spectrum disorder was $17 \%(95 \% \mathrm{Cl} 12,21 ; n=6$ studies $)$ and attention deficit hyperactivity disorder $26 \%(95 \% \mathrm{Cl} 17,35 ; \mathrm{n}=7$ studies. Anxiety prevalence was 22\% in DMD (14,30; n=4 studies) and BMD 19\% (13,25; n=3 studies), and depression 14\% in DMD (7,22; $n=3$ studies) and 17\% in BMD (5,30; $n=4$ studies). There was a strong isoform relationship between lack of shorter dystrophin isoforms and increasing prevalence of intellectual disability, as has been shown previously [31] but no clear relationship of isoforms with other neurodevelopmental and emotional problems.

Imaging studies in the brain in DMD: Erik Niks, Leiden, The Netherlands

Dr. Niks presented an overview of the imaging studies that have been performed in DMD. While the first reports on neuroimaging in DMD were obtained with $\mathrm{CT}$, there are now several imaging studies where advanced MRI techniques were used to assess the brain. Dr. Niks first explained the different types of MR techniques that have been used in DMD and BMD, including T1-weighted imaging for volumetric assessment, diffusion tensor imaging for white matter structural connectivity, functional MRI for functional connectivity, MR spectroscopy for assessment of metabolism and arterial spin labelling for measuring cerebral perfusion.

He then showed an overview of the 10 original articles that have been published using advanced MR techniques (reviewed in [40]), followed by a detailed overview of the MRI studies that were started at the Leiden University Medical Center. The crosssectional dataset was obtained in 29 DMD patients and showed no gross 
abnormalities. Only after quantitative assessment, a smaller total brain and smaller grey matter volume were found compared to age-matched healthy controls [18]. When dividing the group into patients who were predicted to miss only Dp427 and those who were predicted to miss both Dp427 and Dp140, there was a clear staircase effect with the healthy controls showing the highest grey matter volumes, the group missing Dp427 showing somewhat lower grey matter volumes and the group missing both isoforms having the lowest grey matter volume. A short neuropsychological examination (NPE) was also performed on the same day as the MRI, and even though there was no direct relation between MRI and NPE, the same staircase phenomenon was present in a composite score for information processing. This study was later expanded by adding a UK cohort from the John Walton Center for Muscular Dystrophy and changing the design to a longitudinal study. In addition, a group of patients missing all dystrophin isoforms was recruited in the UK. When adding cross-sectional data from the UK cohort to this analysis, the same staircase phenomena persisted with the group missing all isoforms at the lower end of the spectrum.

Dr. Niks then proceeded to show results from the diffusion tensor imaging data, which showed lower fractional anisotropy and higher mean diffusivity in DMD compared to healthy controls which corresponds to changes in white mater structure in DMD. He highlighted ongoing research to link reading performance of this cohort to specific white matter tracts known to be involved in reading.

The longitudinal cohort was also assessed using resting state fMRI, which assesses the functional connectivity of the brain without using a specific task in the scanner. There are multiple networks in the brain which are active during resting conditions, and Dr. Niks showed results from an ongoing analysis. 
Assessments of cerebral metabolism using MR spectroscopy and PET have been done in several other cohorts, including phosphorous MRS in 10 DMD boys and agematched controls, where higher inorganic phosphate to ATP, phosphomonoesters and phosphocreatine were reported. Proton MRS has shown conflicting results, where both increased and decreased choline, decreased glutamate and increased N-Acetyl Aspartate levels have been reported in different areas of the brain. The Dutch cohort showed preserved metabolite levels in the three brain regions that were investigated, and increased variance in glutamate in the frontal cortex. A PET study in four DMD patients and a later one in ten DMD patients showed glucose hypometabolism in the right sensorimotor cortex, bilateral medial temporal structures and cerebellar cortex.

Dr. Niks finalized his overview with data on cerebral perfusion and summarizing remarks. Data from the same Dutch study cohort as where reduced grey matter volume was found also showed a $17 \%$ reduction in cerebral blood flow, which was independent of the reduced grey matter volume. In summary, in DMD, there is a clear lower grey matter volume and cerebral blood flow, with altered white matter structure, which are dependent on the location of the mutation on the $D M D$ gene. The relation with the long-term use of steroids, and whether these changes are progressive or amenable to treatment is still unknown. As many of these studies were single site or dual-site studies, the field is in need for larger multi-center studies.

\section{Lessons regarding cognition from the DMD Natural History Study and Clinical} trials to date: Mathula Thangarajh, Richmond, VA

Dr. Thangarajh performed a gap analysis regarding studies focusing on cognition in clinical trials. Cognitive health is considered both a clinical and research unmet need 
in DMD. Although the standard-of-care recommendations recommend screening at the time of diagnosis and periodically thereafter, at least in the United States, physicians report that barriers for implementation include (i) out-of-pocket costs for mental health services, and (ii) access to see a neuropsychologist. Through the CINRG DMD Natural History Study, it was found that more than $80 \%$ of the boys were not screened by a neuropsychologist despite parental concern [41]. These access barriers also affect research priorities. The National Institute of Health's Muscular Dystrophy Coordinating Committee's research priorities include clinical trial readiness. Yet the lack of reliable and robust clinical outcome measures and a welldesigned natural history evaluating cognition are major impediments to progress. The use of technology-enabled tools like the NIH Toolbox (https://www.healthmeasures.net/explore-measurement-systems/nih-toolbox) [42] can allow for an increase in cognitive surveillance in DMD, and measures within the $\mathrm{NIH}$ Toolbox also has promise as a performance outcome measure for clinical trials. Cognitive problems begin in infancy and persist, as has been shown using instruments such as Bayley-III and Griffith scales. Work from the Muscular Dystrophy Surveillance and Tracking Network has shown that speech delay increases resource utilization during school years [43]. Further, grade repetition is common in DMD, and in those with speech delay and academic performance is greatly affected in DMD.

A major gap in this field is also whether cognitive problems in DMD are static versus progressive. There is growing knowledge both through the work of Dr. Hendriksen and unpublished data on digit span that there is developmental stagnation in DMD [21]. Also, there is a clear genotypic risk for working memory deficits and speech delay in DMD. The underlying neural circuitry affected by these genotypes is yet to 
be well-understood. Several clinicians also highlighted that current clinical trials do not include cognition-related measures.

Briefly, there was a discussion on whether we can use home-based cognitive rehabilitation technology such as CogMed (https://www.cogmed.com/) to improve working memory. This area of research is not without its own controversy, but its potential in DMD is yet to be evaluated. Finally, it is becoming more clear that better cognition skills affects health maintenance; an earlier study showed that individuals with DMD with better IQ survived longer [44]. Therefore, the use of standardized tools that can be used in both clinical and research settings would greatly improve this area of unmet need.

The parents' and patients' perspectives: Elizabeth Vroom (United Parent Project Muscular dystrophy, the Netherlands) and Francois Lamy (Telethon, France).

To stress the utmost importance of brain related comorbidities for patients' and parents' quality of life they suggested the idea to change DMD into DND representing Duchenne Neuromuscular Dystrophy. It was stressed that parents need an early and robust diagnoses in order to build proper care. So, they urge clinicians to speak the truth to parents in this respect. Parents further need awareness, consensus about screening and implementation of existing knowledge. It should also be stressed that brain and motor functioning may not be in sync; i.e. while motor function deteriorates, brain function may improve. Different behavioural problems in DMD patients were positively framed: stubbornness reflecting obstinacy; disharmony in cognition reflecting talent; poor working memory being counter parted by good long-term memory and being difficult to handle which may reflect sensitivity. Furthermore, an appeal was held not to leave anyone behind in screening or testing 
on brain related comorbidities: DMD boys with no obvious problems as in low IQ or behavioural issues could still have specific learning issues which - if not diagnosed and supported properly - can hold them back to reach their full potential. In addition, is it allowed to test sisters with a learning problem without a referral question? Finally, a plea for caution was made with respect to misinterpreting normality.

Overview of learning and behaviour issues in DMD and BMD Jos Hendriksen, Kempenhaege, the Netherlands.

Dr. Hendriksen presented the results of a recently published review about the state of the art of assessment tools available to measure behaviour [45]. In the 51 studies reviewed, a total of 61 different instruments were used with none of them being specifically developed for DMD. Furthermore, ten different domains with respect to learning and behaviour problems in DMD were described. The term "big ten of Duchenne" was proposed by Hendriksen describing ten areas of interest: dyslexia and dyscalculia, within the learning domain; automatization, working memory and attention for the neurocognitive domain, attention deficit hyperactivity disorder (ADHD), obsessive compulsive disorder (OCD) and ASD for the neuropsychiatric domain and finally anxiety and depression for the emotional domain [46]. One important issue to cover in diagnosis is that the pattern of comorbidities can be highly variable and illustrated in Figure 1 . The reality of the big ten is a picture of a complex and difficult to understand overlap of these problem areas. Unalloyed problems are rare. One should take care of complexity with overlap, which may hinder adequate assessment. 
Standards of care: Mary Colvin, Massachusetts, USA.

As co-author of a Lancet Neurology publication on management of DMD [47], Dr Colvin summarized five targets for the future to be done. First of all, there is a need for a broader dissemination of knowledge surrounding neuropsychiatric conditions that are frequently comorbid with DMD, so that patients can be appropriately referred for psychiatric services (evaluation and treatment). One important issue to resolve in the near future is to generate a review paper that summarizes the recent neuroimaging and neuropsychological literature, so that it is easier to disseminate the knowledge to practitioners. A second aim would be to facilitate psychosocial care, as there is a need for better screening instruments that can be implemented within the neuromuscular clinic. These instruments should screen for both neuropsychiatric conditions and cognitive/learning difficulties. It was emphasized that screening instruments should not be used in lieu of neuropsychological evaluations. For neuropsychological evaluations, the use of well-validated and comprehensive clinical instruments was strongly recommended over those developed for research (e.g. NIH Toolbox). Interpretation of individual test performances should be performed by psychologists with expertise in assessment given the variability in skills that often characterizes BMD and DMD neuropsychological profiles. A third aim for the next future would be to better understand and study treatment options for DMD, especially medication and therapy. In the fourth place, it was stressed that there has not been enough attention to the developmental course over time and this is an important question given some of the findings from animal studies that raises the possibility of developmental plateau or regression. We need to start publishing longitudinal studies to characterize this (e.g., is there developmental plateau/regression in cognitive skills, does depression emerge later, how does 
insight/cognitive status impact emotional/social functioning). Finally, it was stressed that we need to assess family functioning in the clinic and provide better supportive resources - ideally we would track family functioning using quantitative measures to better understand factors that promote family resilience and improved functioning.

Learning disabilities: Janet Hoskin, London, UK.

Boys with DMD can experience many difficulties associated with developmental dyslexia. These include deficits in phonological discrimination and manipulation which are skills necessary in initial reading, and speech delays which will impact on the development of these skills. More recently, research has shown that boys with DMD score more poorly on tests of rapid naming than their peers indicating a difficulty to retrieve words and phonological codes from long term memory, and possibly a deficit in consolidation of learning. As in dyslexia, many boys with DMD appear to have working memory impairment which can affect their ability to blend and segment phonemes when learning to read and spell, as well as create difficulties in arithmetic. This can also affect their ability to follow verbal instructions. Studies have also reported on a deficit in verbal memory span suggesting that it is the length of information boys with DMD struggle with rather than manipulating information. Others report problems with delayed rather than immediate memory suggesting difficulties with consolidation of learning and executive function. Hinton's study in 2000 suggests a significant 'learning profile' to be present in all boys with DMD regardless of IQ where their knowledge and vocabulary is consistently a strength in comparison to skills involving working memory [48]. 
In practice, schools struggle with the diagnosis and often feel they need permission to intervene with reading and learning skills. This can lead to low expectations and a dominance of the physical diagnosis, and as a consequence boys can be transferred to special school when it is rarely necessary. When teachers are made aware of the child's strong receptive vocabulary they are often surprised. Referrals to a speech and language therapist should be made for those children with speech and language impairment as soon as possible. Best practice pedagogical strategies for reading difficulties should be employed such as a sequential structured and systematic synthetic phonics programme combined with access to books to continue to develop vocabulary and interest in stories. It is particularly important to ensure that appropriate early intervention is put in place as we know that adults with DMD (which are a growing population) have reported regret at the lack of formal qualifications which can impact on future life opportunities [49].

\section{Neurovascular coupling, cognition and effects of sildenafil in BMD patients.} John Vissing, Copenhagen, Denmark.

Dr. Vissing presented data on somatosensory neurovascular coupling in 14 patients with BMD and 10 healthy persons by investigating the blood-oxygen-level-dependent (BOLD) brain response to median nerve stimulation. Patients had smaller BOLD responses and higher electrophysiological activity in the relevant somatosensory cortex compared with healthy controls [50]. This points to an uncoupling between activity and oxygen delivery in the brain of BMD patients. To explore whether this uncoupling could be improved by treatment with the PDE5 inhibitor, sildenafil, 17 patients with BMD were subjected to a cross-over treatment with sildenafil and placebo for 4 weeks each. Sildenafil-treated patients showed increased task-induced BOLD response, while cerebral blood flow and brain activity were unchanged to 
placebo [51]. By contrast, sildenafil failed to influence cardiac function (left ventricular end diastolic volume, exercise induced cardiac output and brachial artery flow) and muscle function (6MWT distance and maximal oxygen uptake) [52]. The explanation for this could be that muscle in BMD lacks PDE5 protein [52].

Treatment of neuropsychiatric comorbidity for DMD and BMD Philipe Collin and Jos Hendriksen, the Netherlands.

Dr. Collin and Dr. Hendriksen presented data on the psychopharmacological treatment options for DMD and BMD, which are a new area of interest which is still in its infancy. Until now, only one study on the effectiveness of methylphenidate in a clinical population of DMD was published [53] and a case report study reporting a clinical significant effect of Fluoxetine in OCD behaviour in DMD [54].

Pharmacotherapy is always the last step in treatment: psychosocial interventions such as counseling and behavior therapy are the first options to consider. Six indications for medication in DMD were described: (i) ADHD, (ii) OCD, (iii) ASD especially with respect to rigidity, (iv) intermittent explosive disorders, (v) depressive complaints and (vi) anxiety disorders. Important side effects to consider are cardiac problems, excessive weight gain and stagnating. Data were presented from the outpatient clinic in Kempenhaeghe where 26 boys with DMD were put on psychopharmaceutic treatment in the last years. Preliminary data show promising effects which need further scientific consideration. It was suggested that a standard operating procedure for treatment of neuropsychiatric comorbidity is needed and that it is important to objectively monitor benefits and side effects. 
Recommendations for registries: Francesco Muntoni, Volker Straub, Ulrike Schara (Germany), Erik Niks

A call for setting up an interactive platform that is able to capture data both from the clinic, school and home was discussed. Such a platform would allow harmonization of data; one suggestion was to use the minimal data set. A systematic collection would improve the lack of information regarding CNS manifestations while also improving awareness regarding cognitive health in DMD.

To make progress, the group came with a "S.M.A.R.T" approach:

S: Step-wise. An approach of selecting an international working group that consists of neuromuscular physicians, neuropsychologists, psychiatrists, educational specialists, parents and advocacy groups. This group will perform literature review, publish and consider tools that can be used for implementation.

M: Model creation. This will allow integrating bench-to-bed side work, and true translational impact of our workshop. A working group will help establish a standard operating procedure of preclinical models which will be used across laboratories. This will decrease variability in data and findings, especially as behavioural studies can be very challenging to be reproduced. While the team agreed this was a good start, there was also discussion to allow for adaptation as new knowledge and approaches will be made. A working document will be prepared and reviewed periodically to incorporate changes.

A: Awareness. The group recognized that awareness creation through advocacy is the biggest impact we can create. To make this methodically, ideas proposed including dedicated hours during World Duchenne Day and dedicated sections in neuromuscular conferences such as World Muscle Society. The BIND project, 
explained below and led by Professor Muntoni could be another platform. The group cannot emphasize enough the role of advocacy groups that have educated clinicians, governments and policy work through their role.

R: Research. Fundamental to incremental progress will be sound research into underlying pathogenesis of CNS manifestations, the role of dystrophin isoforms in the brain, dystrophin protein biology, behavioural studies in animals and in humans, and therapeutic approaches.

T: Trial readiness. The lack of well-designed natural history studies, potential cognitive biomarker(s) and clinical trial outcome measures were addressed. However, the group was optimistic given the tremendous growth of treatment approaches in neurological diseases. Collaborations, collective grant applications and sharing resources were highlighted as steps to clinical trials readiness. 
Workshop participants (ENMC $249^{\text {th }}$ workshop studygroup).

Dr Y. Aoki (Japan), Dr P. Collin (the Netherlands), Dr M. Colvin (USA), Dr N.

Doorenweerd (the Netherlands), Prof A. Ferlini (Italy), Dr A. Goyenvalle (France), Dr J.J. Hendriksen (the Netherlands), Dr J. Hoskin (UK), Dr H.E. Kan (the Netherlands), Mr F. Lamy (France), Dr K. Maresh (UK), Prof F. Muntoni (UK), Dr E.H. Niks (the Netherlands), Prof U. Schara (Germany), Prof D. Skuse (UK), Prof V. Straub (UK), Prof S. Takeda (Japan), Dr M. Thangarajh (USA), Dr N. Truba (USA), Prof S. Tyagarajan (Switzerland), Dr C. Vaillend (France), Dr M. van Putten (the Netherlands), Prof J. Vissing (Denmark), E. Vroom (the Netherlands).

\section{Acknowledgements.}

This Workshop was made possible thanks to the financial support of the European Neuromuscular Centre (ENMC) and ENMC main sponsors: Association Française centre les Myopathies (France), Deutsche Gesellschaft für Muskelkranke (Germany), Muscular Dystrophy Campaign (UK), Muskelsvindfonden (Denmark), Prinses Beatrix Spierfonds (The Netherlands), Schweizerische Stiftung für die Erforschung der Muskelkrankheiten (Switzerland), Telethon Foundation (Italy), Spierziekten Nederland (The Netherlands), and Associated members: Finnish Neuromuscular Association (Finland). The authors and a number of participants in the workshop are members of the European Reference Network for Rare Neuromuscular Diseases [ERNE EURO-NMD].

The workshops participants are also grateful for the financial support of Parent Project Muscular Dystrophy who made the participation of colleagues from USA possible. 
The participants' agreed to collaborate further regarding patient registries, preclinical models, and clinical studies on these topics and working groups were formed to address specific areas. This collaboration will also be facilitated by the recently awarded European Union H2020 grant to address the Brain Involvement iN Dystrophinopathies (BIND) which has started in January 2020. 


\section{References.}

[1] Birnkrant DJ, Bushby K, Bann CM, Apkon SD, Blackwell A, Colvin MK, et al. Diagnosis and management of Duchenne muscular dystrophy, part 3: primary care, emergency management, psychosocial care, and transitions of care across the lifespan. Lancet Neurol 2018;17:445-455.

[2] Muntoni F, Torelli S, Ferlini A. Dystrophin and mutations: one gene, several proteins, multiple phenotypes. Lancet Neurol 2003;2:731-40.

[3] Nudel U, Zuk D, Einat P, Zeelon E, Levy Z, Neuman S, et al. Duchenne muscular dystrophy gene product is not identical in muscle and brain. Nature 1989;337:76-8.

[4] Monaco AP, Neve RL, Colletti-Feener C, Bertelson CJ, Kurnit DM, Kunkel LM. Isolation of candidate cDNAs for portions of the Duchenne muscular dystrophy gene. Nature 1986;323:646-50.

[5] Gorecki DC, Monaco AP, Derry JM, Walker AP, Barnard EA, Barnard PJ. Expression of four alternative dystrophin transcripts in brain regions regulated by different promoters. Hum Mol Genet 1992;1:505-10.

[6] D'Souza VN, Nguyen TM, Morris GE, Karges W, Pillers DA, Ray PN. A novel dystrophin isoform is required for normal retinal electrophysiology. Hum Mol Genet 1995;4:837-42.

[7] Lidov HG, Kunkel LM. Dp140: alternatively spliced isoforms in brain and kidney. Genomics 1997;45:132-9.

[8] Byers TJ, Lidov HG, Kunkel LM. An alternative dystrophin transcript specific to peripheral nerve. Nat Genet 1993;4:77-81. 
[9] Austin RC, Howard PL, D'Souza VN, Klamut HJ, Ray PN. Cloning and characterization of alternatively spliced isoforms of Dp71. Hum Mol Genet 1995;4:1475-83.

[10] Tinsley JM, Blake DJ, Davies KE. Apo-dystrophin-3: a 2.2kb transcript from the DMD locus encoding the dystrophin glycoprotein binding site. Hum Mol Genet 1993;2:521-4.

[11] Fratter C, Dalgleish R, Allen SK, Santos R, Abbs S, Tuffery-Giraud S, et al. EMQN best practice guidelines for genetic testing in dystrophinopathies. Eur $\mathrm{J}$ Hum Genet 2020.

[12] Gazzoli I, Pulyakhina I, Verwey NE, Ariyurek Y, Laros JF, t Hoen PA, et al. Non-sequential and multi-step splicing of the dystrophin transcript. RNA Biol 2016;13:290-305.

[13] Lidov HG, Selig S, Kunkel LM. Dp140: a novel 140 kDa CNS transcript from the dystrophin locus. Hum Mol Genet 1995;4:329-35.

[14] Spitali P, van den Bergen JC, Verhaart IE, Wokke B, Janson AA, van den Eijnde R, et al. DMD transcript imbalance determines dystrophin levels. FASEB J 2013;27:4909-16.

[15] Gherardi S, Bovolenta M, Passarelli C, Falzarano MS, Pigini P, Scotton C, et al. Transcriptional and epigenetic analyses of the DMD locus reveal novel cisacting DNA elements that govern muscle dystrophin expression. Biochim Biophys Acta Gene Regul Mech 2017;1860:1138-1147.

[16] Gallia GL, Zhang M, Ning Y, Haffner MC, Batista D, Binder ZA, et al. Genomic analysis identifies frequent deletions of Dystrophin in olfactory neuroblastoma. Nat Commun 2018;9:5410. 
[17] Doorenweerd N, Mahfouz A, van Putten M, Kaliyaperumal R, PAC TH, Hendriksen JGM, et al. Timing and localization of human dystrophin isoform expression provide insights into the cognitive phenotype of Duchenne muscular dystrophy. Sci Rep 2017;7:12575.

[18] Doorenweerd N, Straathof CS, Dumas EM, Spitali P, Ginjaar IB, Wokke BH, et al. Reduced cerebral gray matter and altered white matter in boys with Duchenne muscular dystrophy. Ann Neurol 2014;76:403-11.

[19] Aranmolate A, Tse N, Colognato $\mathrm{H}$. Myelination is delayed during postnatal brain development in the mdx mouse model of Duchenne muscular dystrophy. BMC Neurosci 2017;18:63.

[20] Patel AM, Wierda K, Thorrez L, van Putten M, De Smedt J, Ribeiro L, et al. Dystrophin deficiency leads to dysfunctional glutamate clearance in iPSC derived astrocytes. Transl Psychiatry 2019;9:200.

[21] Hellebrekers DMJ, Doorenweerd N, Sweere DJJ, van Kuijk SMJ, AartsmaRus AM, Klinkenberg S, et al. Longitudinal follow-up of verbal span and processing speed in Duchenne muscular dystrophy. Eur J Paediatr Neurol 2020;25:120-126.

[22] Waite A, Brown SC, Blake DJ. The dystrophin-glycoprotein complex in brain development and disease. Trends Neurosci 2012;35:487-96.

[23] Sekiguchi M, Zushida K, Yoshida M, Maekawa M, Kamichi S, Yoshida M, et al. A deficit of brain dystrophin impairs specific amygdala GABAergic transmission and enhances defensive behaviour in mice. Brain 2009;132:124-35. 
[24] Vaillend C, Chaussenot R. Relationships linking emotional, motor, cognitive and GABAergic dysfunctions in dystrophin-deficient mdx mice. Hum Mol Genet 2017;26:1041-1055.

[25] Miranda R, Sebrie C, Degrouard J, Gillet B, Jaillard D, Laroche S, et al. Reorganization of inhibitory synapses and increased PSD length of perforated excitatory synapses in hippocampal area CA1 of dystrophin-deficient $\mathrm{mdx}$ mice. Cereb Cortex 2009;19:876-88.

[26] Dallerac G, Perronnet C, Chagneau C, Leblanc-Veyrac P, Samson-Desvignes N, Peltekian E, et al. Rescue of a dystrophin-like protein by exon skipping normalizes synaptic plasticity in the hippocampus of the mdx mouse. Neurobiol Dis 2011;43:635-41.

[27] Goyenvalle A, Griffith G, Babbs A, El Andaloussi S, Ezzat K, Avril A, et al. Functional correction in mouse models of muscular dystrophy using exonskipping tricyclo-DNA oligomers. Nat Med 2015;21:270-5.

[28] Vaillend C, Perronnet C, Ros C, Gruszczynski C, Goyenvalle A, Laroche S, et al. Rescue of a dystrophin-like protein by exon skipping in vivo restores GABAA-receptor clustering in the hippocampus of the mdx mouse. Mol Ther 2010;18:1683-8.

[29] Daoud F, Candelario-Martinez A, Billard JM, Avital A, Khelfaoui M, Rozenvald $\mathrm{Y}$, et al. Role of mental retardation-associated dystrophin-gene product Dp71 in excitatory synapse organization, synaptic plasticity and behavioral functions. PLoS One 2008;4:e6574.

[30] Chaussenot R, Amar M, Fossier P, Vaillend C. Dp71-Dystrophin Deficiency Alters Prefrontal Cortex Excitation-Inhibition Balance and Executive Functions. Mol Neurobiol 2019;56:2670-2684. 
[31] Ricotti V, Mandy WP, Scoto M, Pane M, Deconinck N, Messina S, et al. Neurodevelopmental, emotional, and behavioural problems in Duchenne muscular dystrophy in relation to underlying dystrophin gene mutations. Dev Med Child Neurol 2016;58:77-84.

[32] Miranda R, Nudel U, Laroche S, Vaillend C. Altered presynaptic ultrastructure in excitatory hippocampal synapses of mice lacking dystrophins Dp427 or Dp71. Neurobiol Dis 2011;43:134-41.

[33] Remmelink E, Aartsma-Rus A, Smit AB, Verhage M, Loos M, van Putten M. Cognitive flexibility deficits in a mouse model for the absence of full-length dystrophin. Genes Brain Behav 2016;15:558-67.

[34] Miranda R, Laroche S, Vaillend C. Reduced neuronal density in the CA1 anterodorsal hippocampus of the mdx mouse. Neuromuscul Disord 2016;26:775-781.

[35] Relizani K, Griffith G, Echevarria L, Zarrouki F, Facchinetti P, Vaillend C, et al. Efficacy and Safety Profile of Tricyclo-DNA Antisense Oligonucleotides in Duchenne Muscular Dystrophy Mouse Model. Mol Ther Nucleic Acids 2017;8:144-157.

[36] Aupy P, Zarrouki F, Sandro Q, Gastaldi C, Buclez PO, Mamchaoui K, et al. Long-Term Efficacy of AAV9-U7snRNA-Mediated Exon 51 Skipping in mdx52 Mice. Mol Ther Methods Clin Dev 2020;17:1037-1047.

[37] Bagdatlioglu E, Porcari P, Greally E, Blamire AM, Straub VW. Cognitive impairment appears progressive in the $\mathrm{mdx}$ mouse. Neuromuscul Disord 2020;30:368-388.

[38] Ennaceur A, Delacour J. A new one-trial test for neurobiological studies of memory in rats. 1: Behavioral data. Behav Brain Res 1988;31:47-59. 
[39] Cotton S, Voudouris NJ, Greenwood KM. Intelligence and Duchenne muscular dystrophy: full-scale, verbal, and performance intelligence quotients. Dev Med Child Neurol 2001;43:497-501.

[40] Doorenweerd N. Combining genetics, neuropsychology and neuroimaging to improve understanding of brain involvement in Duchenne muscular dystrophy - a narrative review. Neuromuscul Disord 2020;30:437-442.

[41] Thangarajh M, Spurney CF, Gordish-Dressman H, Clemens PR, Hoffman EP, McDonald CM, et al. Neurodevelopmental Needs in Young Boys with Duchenne Muscular Dystrophy (DMD): Observations from the Cooperative International Neuromuscular Research Group (CINRG) DMD Natural History Study (DNHS). PLoS Curr 2018;10.

[42] Thangarajh M, Kaat AJ, Bibat G, Mansour J, Summerton K, Gioia A, et al. The NIH Toolbox for cognitive surveillance in Duchenne muscular dystrophy. Ann Clin TransI Neurol 2019;6:1696-1706.

[43] Soim A, Lamb M, Campbell K, Pandya S, Peay H, Howard Jr JF, et al. A cross-sectional study of school experiences of boys with Duchenne and Becker muscular dystrophy. Physical Disabilities: Education and Related Services 2016;35:1-22.

[44] Miller G, Tunnecliffe M, Douglas PS. IQ, prognosis and Duchenne muscular dystrophy. Brain Dev 1985;7:7-9.

[45] Hellebrekers DMJ, Lionarons JM, Faber CG, Klinkenberg S, Vles JSH, Hendriksen JGM. Instruments for the Assessment of Behavioral and Psychosocial Functioning in Duchenne and Becker Muscular Dystrophy; a Systematic Review of the Literature. J Pediatr Psychol 2019;44:1205-1223. 
[46] Hendriksen RGF, Vles JSH, Aalbers MW, Chin RFM, Hendriksen JGM. Brainrelated comorbidities in boys and men with Duchenne Muscular Dystrophy: A descriptive study. Eur J Paediatr Neurol 2018;22:488-497.

[47] Birnkrant DJ, Bushby K, Bann CM, Apkon SD, Blackwell A, Brumbaugh D, et al. Diagnosis and management of Duchenne muscular dystrophy, part 1: diagnosis, and neuromuscular, rehabilitation, endocrine, and gastrointestinal and nutritional management. Lancet Neurol 2018;17:251-267.

[48] Hinton VJ, De Vivo DC, Nereo NE, Goldstein E, Stern Y. Poor verbal working memory across intellectual level in boys with Duchenne dystrophy. Neurology 2000;54:2127-32.

[49] Rahbek J, Werge B, Madsen A, Marquardt J, Steffensen BF, Jeppesen J. Adult life with Duchenne muscular dystrophy: observations among an emerging and unforeseen patient population. Pediatr Rehabil 2005;8:17-28.

[50] Lindberg U, Kruuse C, Witting N, Jorgensen SL, Vissing J, Rostrup E, et al. Altered somatosensory neurovascular response in patients with Becker muscular dystrophy. Brain Behav 2018;8:e00985.

[51] Lindberg U, Witting N, Jorgensen SL, Vissing J, Rostrup E, Larsson HB, et al. Effects of Sildenafil on Cerebrovascular Reactivity in Patients with Becker Muscular Dystrophy. Neurotherapeutics 2017;14:182-190.

[52] Witting N, Kruuse C, Nyhuus B, Prahm KP, Citirak G, Lundgaard SJ, et al. Effect of sildenafil on skeletal and cardiac muscle in Becker muscular dystrophy. Ann Neurol 2014;76:550-7.

[53] Lionarons JM, Hellebrekers DMJ, Klinkenberg S, Faber CG, Vles JSH, Hendriksen JGM. Methylphenidate use in males with Duchenne muscular 
dystrophy and a comorbid attention-deficit hyperactivity disorder. Eur $\mathrm{J}$ Paediatr Neurol 2019;23:152-157.

[54] Hendriksen JG, Klinkenberg S, Collin P, Wong B, Niks EH, Vles JS. Diagnosis and treatment of obsessive compulsive behavior in a boy with Duchenne muscular dystrophy and autism spectrum disorder: A case report.

Neuromuscul Disord 2016;26:659-661. 
Table and figure legends

Table 1. Organisation of the dystrophin locus including its various isoforms .

Figure 1. The big ten of Duchenne and patterns of comorbidities.

Ten areas of interest, divided in four domains. Learning domain; green, neurocognitive domain; red, neuropsychiatric domain; black and emotional domain; orange. 